

\title{
Bell, Group and Tangle
}

\author{
Allan Solomon \\ Department of Physics, Open University, UK \\ and \\ LPTMC, University of Paris VI, France
}

\begin{abstract}
The "Bell" of the title refers to bipartite Bell states, and their extensions to, for example, tripartite systems. The "Group" of the title is the Braid Group in its various representations; while "Tangle" refers to the property of entanglement which is present in both of these scenarios. The objective of this note is to explore the relation between Quantum Entanglement and Topological Links, and to show that the use of the language of entanglement in both cases is more than one of linguistic analogy.
\end{abstract}

PACS numbers: $03.65 . \mathrm{Ud}$, 03.65.Fd

Entanglement is to Quantum Theory what Number Theory is to Mathematics: - The subject is fundamental and the problems are easily understood - but the solutions are elusive.

\section{INTRODUCTION}

The objective of this talk is to introduce the concept of Quantum Entanglement as well as some elements of Topological Entanglement such as braids and links, and explore the relation between these ideas.

Although bipartite entanglement has been well analyzed, with useful and easily-computed measures available, extending the analysis beyond the two-subspace regime is currently an open problem.

In this note we first explore the problem of extending the Von Neumann entropy-type of entanglement measure to a tripartite system, showing how a naïve extension fails.

We relate this case to the theory of links, and on the basis of this analogy introduce the concept of Braid Groups.

We then discuss the relation between braid representations and unitary entanglement-producing operators in quantum mechanics, illustrating by the examples of the Hopf Link as a quantum entangled bipartite system, and the Borromean Rings as an entangled tripartite system. 


\section{QUANTUM ENTANGLEMENT}

\section{A. Vector Spaces and Entanglement}

A basic operation for vectors is addition. For mathematicians therefore, vector addition presents no surprises. For physicists, vector addition is such a remarkable property that in quantum mechanics the phenomena it gives rise to it go by many names, superposition rule, interference, entanglement, ...

Entanglement is a property of the vectors in direct product spaces, the simplest case being that of a bipartite space, $V_{1} \otimes V_{2}$. If the space $V_{1}$ has basis $\left\{v_{i}^{1}: i=1 \ldots m\right\}$ and $V_{2}$ basis $\left\{v_{j}^{2}: j=1 \ldots n\right\}$ then $V_{1} \otimes V_{2}$ has basis $\left\{v_{i}^{1} \otimes v_{j}^{2}: i=1 \ldots n, j=1 \ldots n\right\}$.

Since a vector of $V_{1} \otimes V_{2}$ is a sum of products of basis vectors of $V_{1}$ and $V_{2}$, it need not necessarily be itself a product of a vector of $V_{1}$ and a vector of $V_{2}$. If it is not, we say that it is an entangled vector.

In this note we shall take our examples from 2-spaces i.e. whose elements are qubits.

Example II.1 Take each of the vector spaces $V_{1}, V_{2}$ as 2-dimensional with basis

$$
e_{1}=|0\rangle=\left[\begin{array}{l}
1 \\
0
\end{array}\right] \quad e_{2}=|1\rangle=\left[\begin{array}{l}
0 \\
1
\end{array}\right]
$$

then $e_{1} \otimes e_{2}+e_{2} \otimes e_{2}=\left(e_{1}+e_{2}\right) \otimes e_{2}=|0,1\rangle+|1,1\rangle$ is factorizable , therefore not entangled,or sometimes called separable; while $e_{1} \otimes e_{2}+e_{2} \otimes e_{1}$ is entangled.

\section{B. Local transformations}

Although sometimes referred to as a "resource", entanglement is rather peculiar in that it is not invariant under unitary transformations.

Example II.2 Consider the unitary transformation $U \equiv \frac{1}{\sqrt{2}}\left[\begin{array}{cccc}1 & 0 & 0 & -1 \\ 0 & 1 & -1 & 0 \\ 0 & 1 & 1 & 0 \\ 1 & 0 & 0 & 1\end{array}\right]$.

One easily evaluates $U|0,0\rangle=\frac{1}{\sqrt{2}}(|0,0\rangle+|1,1\rangle)$ which is a (intuitively, maximally) entangled state (Bell state).

The situation is different for local transformations: 
Definition II.3 A local unitary transformation $U$ on the bipartite space $V_{A} \otimes V_{B}$ is one of the form $U_{A} \otimes U_{B}$, where $U_{A}$ (resp. $\left.U_{B}\right)$ is a unitary transformation on $V_{A}$ (resp. $V_{B}$ ).

Clearly, a local unitary transformation leaves a factorizable state factorized. Conversely, entangled states remain entangled - since (local) unitary transformations are invertible (with local inverses). More generally, it can be shown that local transformations leave measures of entanglement, such as that introduced in section(IID), invariant.

\section{States, pure and mixed}

\section{Pure States}

Vectors correspond to pure states. For example $|\psi\rangle=\sum_{i}|i\rangle$ or $\left[\begin{array}{l}\alpha \\ \beta\end{array}\right]=\alpha\left[\begin{array}{l}1 \\ 0\end{array}\right]+\beta\left[\begin{array}{l}0 \\ 1\end{array}\right]$. We may equally represent a pure state $|\psi\rangle$ by the Operator (Projector) $|\psi\rangle\langle\psi|$ which projects onto that state. In the vector form above, the state $\rho$ is represented by a matrix:

$$
\rho=\left[\begin{array}{l}
\alpha \\
\beta
\end{array}\right]\left[\begin{array}{ll}
\alpha^{*} & \beta^{*}
\end{array}\right]=\left[\begin{array}{cc}
\alpha \alpha^{*} & \alpha \beta^{*} \\
\beta \alpha^{*} & \beta \beta^{*}
\end{array}\right] \quad \rho^{2}=\rho .
$$

NOTE: trace $\rho=1$ (Normalization) and $\rho$ is Positive i.e. $\rho$ is a Hermitian matrix with (semi- ) positive eigenvalues (for a pure state the only non-zero eigenvalue is 1 ).

\section{Mixed States}

We take the preceding properties as our general definition of a (mixed) state; that is,

Definition II.4 $\rho$ is a state if it is a positive matrix of trace 1.

Note: One may readily show that $\rho$ is a (convex) sum of pure states (not a unique sum). A general state is also referred to as a density matrix.

\section{Measures of Entanglement}

\section{Entropy of a state (Von Neumann entropy)}

Definition II.5 The Entropy of the state $\rho$ is given by $E(\rho)=-\operatorname{tr}(\rho \log \rho)=-\sum_{i} \lambda_{i} \log \left(\lambda_{i}\right)$

For qubits we conventionally take logs to base 2 . 


\section{Example II.6 Pure state}

Every pure state has entropy zero. Since every pure (qubit) state is unitarily equivalent to $\rho=$ $\left[\begin{array}{ll}1 & 0 \\ 0 & 0\end{array}\right]$ the entropy is $E(\rho)=1 \log 1+0 \log 0=0$.

\section{Example II.7 Mixed state}

For a general qubit (unitarily equivalent to)

$$
\rho=\left[\begin{array}{cc}
\lambda_{1} & 0 \\
0 & \lambda_{2}
\end{array}\right] \quad \lambda_{1} \geq 0 \quad \lambda_{2} \geq 0 \quad \lambda_{1}+\lambda_{2}=1
$$

we have $E(\rho)=-\lambda_{1} \log \left(\lambda_{1}\right)-\lambda_{2} \log \left(\lambda_{2}\right)$ where we may express the entropy in terms of a single parameter $\lambda(0 \leq \lambda \leq 1) E(\rho)=-\lambda \log \lambda-(1-\lambda) \log (1-\lambda)$. It is easy to show that $0 \leq E(\rho) \leq 1$ with maximum entropy value 1 for $\lambda=1 / 2$ - defining a maximally random state (taking logs to base 2).

\section{Intuitive Measures of Entanglement}

Intuitively a satisfactory measure of the amount of entanglement $\mathcal{E}$ for a two-qubit bipartite system should satisfy the criteria of the following table (for pure states):

\begin{tabular}{|l|c|c|}
\hline State & Entangled? & Entanglement measure $\mathcal{E}$ \\
\hline$(1)$ State $\frac{1}{\sqrt{2}}(|0,0\rangle+|0,1\rangle)$ & No & 0 \\
\hline$(2)$ Bell State $\frac{1}{\sqrt{2}}(|0,0\rangle+|1,1\rangle)$ & Yes & 1 \\
\hline$(3)$ State $\sqrt{\lambda}(|0,0\rangle+\sqrt{1-\lambda}|1,1\rangle)$ & Yes & $0 \leq \mathcal{E} \leq 1$ \\
\hline
\end{tabular}

It turns out that the (Von Neumann) Entropy gives a measure of entanglement for pure states; but not directly, as all pure states have entropy zero.

We must first take the Partial Trace over one subsystem of the bipartite system.

\section{Partial Trace}

Definition II.8 If $V=V_{A} \otimes V_{B}$ then $\operatorname{tr}_{B}\left(Q_{A} \otimes Q_{B}\right)=Q_{A} \operatorname{tr}\left(Q_{B}\right)$. Extend to sums by linearity.

For example, if $Q_{A}=\left|u_{1}\right\rangle\left\langle u_{2}\left|\quad Q_{B}=\right| v_{1}\right\rangle\left\langle v_{2}\right| \quad$ then $\quad \operatorname{tr}_{B}\left(Q_{A} \otimes Q_{B}\right)=|u 1\rangle\langle u 2|\langle v 2 \mid v 1\rangle$.

\section{Example II.9 Non-entangled state}


The density matrix corresponding to $|\alpha\rangle=\frac{1}{\sqrt{2}}(|0,0\rangle+|0,1\rangle)$ is

$$
\begin{gathered}
\rho_{\alpha}=\frac{1}{2}(|0,0\rangle+|0,1\rangle)(\langle 0,0|+\langle 0,1|)=\left[\begin{array}{cccc}
1 / 2 & 1 / 2 & 0 & 0 \\
1 / 2 & 1 / 2 & 0 & 0 \\
0 & 0 & 0 & 0 \\
0 & 0 & 0 & 0
\end{array}\right] \\
\operatorname{tr}_{B}\left(\rho_{\alpha}\right)=\frac{1}{2}(|0\rangle\langle 0|+| 0\rangle\langle 0|)=\left[\begin{array}{cc}
1 & 0 \\
0 & 0
\end{array}\right]
\end{gathered}
$$

The resulting partially-traced state is a pure state, which has entropy zero.

\section{Example II.10 Maximally entangled state}

Consider the (intuitively, maximally) entangled state (Bell State):

$$
\begin{aligned}
|\beta\rangle & =(1 / \sqrt{2})(|0,0\rangle+|1,1\rangle) \\
\rho_{\beta} & =(1 / 2)(|0,0\rangle+|1,1\rangle)(\langle 0,0|+\langle 1,1|) \\
\operatorname{tr}_{B}\left(\rho_{\beta}\right) & =(1 / 2)(|0\rangle\langle 0|+| 1\rangle\langle 1|) \\
& =\left[\begin{array}{cc}
1 / 2 & 0 \\
0 & 1 / 2
\end{array}\right]
\end{aligned}
$$

The resulting (reduced) density matrix has maximum entropy 1 .

\section{Example II.11 Entangled state}

Consider the entangled state :

$$
\begin{aligned}
|\gamma\rangle & =(1 / \sqrt{3})(|0,0\rangle+|0,1\rangle)+|1,0\rangle) \\
\rho_{\gamma} & =(1 / 3)(|0,0\rangle+|0,1\rangle)+|1,0\rangle)(\langle 0,0|+\langle 0,1|+\langle 1,0|) \\
\operatorname{tr}_{B}\left(\rho_{\gamma}\right) & =(1 / 2)(|0\rangle\langle 0|+| 1\rangle\langle 1|) \\
& =\left[\begin{array}{cc}
2 / 3 & 1 / 3 \\
1 / 3 & 1 / 3
\end{array}\right]
\end{aligned}
$$

The resulting (reduced) density matrix has entropy 0.55 .

\section{Example II.12 Interpolating entangled state}

Consider the pure state interpolating between entangled and non-entangled states :

$$
|\Theta\rangle=\cos \theta(|0,0\rangle+\sin \theta|1,1\rangle) .
$$


The reduced density matrix is $\left[\begin{array}{cc}\cos ^{2} \theta & 0 \\ 0 & \sin ^{2} \theta\end{array}\right]$

The corresponding entanglement measure varies from 0 for $\theta=0, \pi / 2$ via a maximum of 1 for $\theta=\pi / 4$ (Bell state).

\section{Entanglement of Formation}

The previous examples indicate that using the entropy of the reduced density matrix gives a useful measure of entanglement for pure states. It can be shown that partially tracing over either subspace for a bipartite system gives the same result. Therefore we may define:

Definition II.13 The measure of entanglement (sometimes referred to as entanglement of formation) for a bipartite pure state is the average of the entropies of the two reduced density matrices (that is, partially traced over each of the two subsystems).

Although a general state is not a unique sum of pure states, we may define:

Definition II.14 The entanglement $\mathcal{E}(\rho)$ of a mixed bipartite state $\rho \in V_{A} \otimes V_{B}$ is given by $\mathcal{E}(\rho)=\min \left\{\sum_{i} \lambda_{i} \mathcal{E}\left(\psi_{i}\right) \mid \rho=\sum_{i} \lambda_{i} \psi_{i}\right\}$ where the $\psi_{i}$ are pure states in $V_{A} \otimes V_{B}$.

The foregoing calculation involves taking the minimum of an infinite set; however, it has been shown that in the case of bipartite states the entanglement (as defined herein) may be obtained from an equally appropriate measure of entanglement, called the concurrence[1]; and this latter is obtainable as a simple function of the eigenvalues of the $4 \times 4$ matrix $\rho$.

The above definition (II.14) extends readily to multipartite mixed states; we thus concentrate in this note on describing entanglement measures for multipartite pure states.

Thus encouraged, the problem now remains to define a measure of entanglement for tripartite (and higher) states.

\section{E. Tripartite states}

Since in the bipartite case one obtains the entanglement by tracing out each space and then averaging, it would seem appropriate in the tripartite case to define the entanglement measure as the average of the three bipartite entanglements obtained by tracing out each of the three subspaces in turn. To see how this naïve approach would work, consider the following example: 


\section{Example II.15}

$$
\begin{aligned}
|\psi\rangle & =\frac{1}{\sqrt{3}}(|1,0,0\rangle+|0,1,0\rangle+|0,0,1\rangle) \\
\rho_{\psi} & =\frac{1}{3}(|1,0,0\rangle+|0,1,0\rangle+|0,0,1\rangle)(\langle 1,0,0|+\langle 0,1,0|+\langle 0,0,1|)
\end{aligned}
$$

Due to the symmetry of this state, the three partial traces are equal, each giving the matrix

$$
\left[\begin{array}{cccc}
1 / 3 & 0 & 0 & 0 \\
0 & 1 / 3 & 1 / 3 & 0 \\
0 & 1 / 3 & 1 / 3 & 0 \\
0 & 0 & 0 & 0
\end{array}\right] .
$$

At this point it would therefore seem reasonable to define an entanglement measure as the average of the three (equal) bipartite measures. In this case the concurrence of each reduced density matrix is $2 / 3$, giving an average concurrence of $2 / 3$, corresponding to an entanglement of .55 , which seems reasonable enough.

However, the "success" of this approach is short-lived, as the next example shows.

We consider the following tripartite analogue of a Bell state (often referred to as a GHZ state[2]), which we intuitively expect to be maximally entangled.

\section{Example II.16}

$$
\begin{aligned}
|\Psi\rangle & =\frac{1}{\sqrt{2}}(|0,0,0\rangle+|1,1,1\rangle) \\
\rho_{\Psi} & =\frac{1}{2}(|0,0,0\rangle+|1,1,1\rangle)(\langle 0,0,0|+\langle 1,1,1|)
\end{aligned}
$$

Again this state has three equal partial traces, each giving the matrix

$$
\left[\begin{array}{cccc}
1 / 2 & 0 & 0 & 0 \\
0 & 0 & 0 & 0 \\
0 & 0 & 0 & 0 \\
0 & 0 & 0 & 1 / 2
\end{array}\right]
$$

which corresponds to a separable (non-entangled) state (concurrence=0). This is certainly not what we intuitively expect for the state $|\Psi\rangle$.

This situation, where we have three subspaces clearly linked (entangled) but each projected subspace is not linked, mirrors the well-known topological feature of the Borromean Rings (see Figure 1). Here we see that all the links are certainly what one reasonably term entangled; however, if 
we remove any link the resulting two are no longer entangled. From the analogy with the previous quantum example it would therefore seem profitable to explore the topological properties of links, and see if we can relate these to the unitary transformations which produce entanglement.

\section{BRAIDS, KNOTS AND LINKS}

In this section we explore braid groups[3], as introduced by Artin[4], considering them as a generalisation of the better known symmetric groups, and their relation to links.

\section{A. Symmetry Group}

The symmetry group $S_{n}$ (sometimes called the permutation group) is defined as the the set of $n$ ! permutations on $n$ distinct objects, combining according to the rule illustrated by

$$
\left(\begin{array}{llll}
1 & 2 & 3 & 4 \\
3 & 1 & 2 & 4
\end{array}\right)\left(\begin{array}{llll}
1 & 2 & 3 & 4 \\
1 & 3 & 2 & 4
\end{array}\right)=\left(\begin{array}{llll}
1 & 2 & 3 & 4 \\
2 & 1 & 3 & 4
\end{array}\right)
$$

for the case of $S_{4}$. A diagrammatic representation of the resultant permutation is found in Figure 2. The symmetric group $S_{n}$ has a presentation in terms of $n-1$ adjacent transpositions ${ }^{1},\left\{s_{i} i=\right.$ $1 \ldots n-1\}$ where $s_{1}$ sends the $i$ to $i+1$ and $i+1$ to $i$. This rather mysterious presentation is:

$$
\begin{aligned}
s_{i} s_{j} & =s_{j} s_{i} \quad|i-j|>1 \\
s_{i} s_{i} & =I \\
s_{i} s_{i+1} s_{i} & =s_{i+1} s_{i} s_{i+1}
\end{aligned}
$$

where Eq.20 plays an important role in the generalization to the Braid group, in which context it is known as the braiding relation or the Yang-Baxter condition.

The foregoing presentation can be implemented by the $n \times n$ matrix representation:

$$
\left[\begin{array}{cccccc}
1 & 0 & 0 & 0 & 0 & 0 \\
0 & 1 & 0 & 0 & 0 & 0 \\
& & \ddots & & & \\
& & & s & & \\
& & & & & \\
& & & & \ddots & \\
0 & 0 & 0 & 0 & 0 & 1
\end{array}\right]
$$

\footnotetext{
${ }^{1}$ The right-hand side of Eq.(17) is an adjacent transposition.
} 
where $s$ is the $2 \times 2$ matrix $\left[\begin{array}{ll}0 & 1 \\ 1 & 0\end{array}\right]$ whose $(1,1)$ element is in the $(i, i)$ position of the matrix representing $s_{i},(i=1 \ldots n-1)$.

\section{B. Braid group}

The braid group is like the symmetric group, but in three dimensions, so you must imagine the arrow joining the elements of the permuted set to go "over" or "under" each other. A diagrammatic representation of the elements $\sigma_{1}$ and $\sigma_{1}^{-1}$ of $B_{4}$ is given by Figure 3. Since now clearly $\sigma_{1}^{2} \neq 1$, all the (non-trivial) Braid groups are infinite dimensional. Just as for the symmetric group, the braid group $B_{n}$ has a presentation in terms of $n-1$ generators $\sigma_{i}$ (and their inverses). This presentation is:

$$
\begin{aligned}
\sigma_{i} \sigma_{j} & =\sigma_{j} \sigma_{i} \quad|i-j|>1 \\
\sigma_{i} \sigma_{i+1} \sigma_{i} & =\sigma_{i+1} \sigma_{i} \sigma_{i+1}
\end{aligned}
$$

where notably the analogue of Eq.(19) is absent. Eq.(22) is known as the braiding relation or the Yang-Baxter condition, as was noted above.

\section{Knots and Links}

Of particular interest to us is the fact that, as shown by Alexander[5], all knots and links may be obtained from elements of a braid group by the simple expedient of joining the the "dots"; that is, join 1 to 1,2 to 2 , and so on.

Example III.1 For the braid group $B_{2}$ with one generator $\sigma_{1}$, in Figure 4 we can see that performing this action with $\sigma_{1}$ gives the unknot.

Example III.2 Similarly, $\sigma_{1}^{2}$ in the braid group $B_{2}$ gives the Hopf Link, as in Figure 5.

More complicated examples are the Olympic Symbol, Figure 6 and the Borromean Rings, as previously noted, Figure 8.

\section{UNITARY REPRESENTATIONS OF BRAID GROUPS AND ENTANGLEMENT}

In order to relate the action of the braid group to unitary transformations on quantum systems, we shall associate each initial point of the braid group description as, for example, in Figure 3, with 
a qubit. A generic unitary representation of the braid group which satisfies the relation Eq.(21) can in principle be obtained from the following:

$$
\hat{\sigma}_{i}=I \times \cdots \times U \times I \cdots \times I
$$

where $I=\left[\begin{array}{ll}1 & 0 \\ 0 & 1\end{array}\right]$ and $U$ is a $4 \times 4$ unitary matrix occupying the $(i, i+1)$ position in the product. Of course it is more difficult to satisfy Eq.(22), the braiding, or Yang-Baxter, relation. We describe one form for $B_{2}$ in the following.

\section{A. Unitary representation for $B_{2}$}

In a sense finding a unitary representation for $B_{2}$ is a trivial exercise, as in this case there are effectively no relations on the single generator $\sigma_{1}$. Thus any unitary matrix will do; but for our purpose we require a $4 \times 4$ unitary matrix - since it is acting on the two-qubit space - and we should like it to mimic the Hopf link; that is, the unitary representative $\hat{\sigma}_{1}^{2}$ should produce a maximally entangled state from a (generic) non-entangled state. We define a (continuous parameter) unitary transformation matrix as follows:

$$
u(\theta)=\left[\begin{array}{cccc}
\cos \theta & 0 & 0 & -\sin \theta \\
0 & \cos \theta & -\sin \theta & 0 \\
0 & \sin \theta & \cos \theta & 0 \\
\sin \theta & 0 & 0 & \cos \theta
\end{array}\right] .
$$

Defining $\hat{\sigma}_{1}=u(\pi / 8)$ so that

$$
\hat{\sigma}_{1}^{2}=U \equiv 1 / \sqrt{2}\left[\begin{array}{cccc}
1 & 0 & 0 & -1 \\
0 & 1 & -1 & 0 \\
0 & 1 & 1 & 0 \\
1 & 0 & 0 & 1
\end{array}\right]
$$

whence one evaluates $U|0,0\rangle=\frac{1}{\sqrt{2}}(|0,0\rangle+|1,1\rangle)$ as in Example(II.2).

The analogous unlinked diagram corresponds (trivially) to $\hat{\sigma}_{1} \hat{\sigma}_{1}^{-1}=I$.

Although this unitary representation produces a maximally entangled (Bell) state from the generic separable state, it is not a true representation of $B_{2}$ since $U^{8}=I$; it is rather a unitary representation of the quasi-symmetric group of the Appendix (Section VII) on one generator, with $R$-exponent $=16$. Further, the unitary operator $u(\theta)$ produces, as expected, a continuous range of entanglements as in Fig.(7); however, the transformation corresponding to $\hat{\sigma}_{1}, u(\pi / 8)$, produces an intermediate value of entanglement, not obviously corresponding to the "unknot". 


\section{B. Unitary representation for $B_{3}$}

Finding unitary representations for $B_{3}$ and beyond is less trivial than for $B_{2}$, since now the braiding relation Eq.(22) must be satisfied. Using $u(\theta)$ as in Eq.(24, we write the representation for $B_{3}$ as

$$
\hat{\sigma}_{1}=u(\theta) \times I, \quad \hat{\sigma}_{2}=I \times u(\theta) .
$$

One calculates that the braiding relation Eq.(22) is satisfied only for $\theta=0, \pi / 4,3 \pi / 4, \pi$ in the range $[0, \pi]$. Choosing the value $\theta=\pi / 4$ gives for $u(\pi / 4)$ the matrix $U$ of Example(II.2). Taking as our paradigm for tripartite entanglement the Borromean Rings of Figure(1) whose braid representation is $\left(\sigma_{1} \sigma_{2}{ }^{-1}\right)^{3}$ from Figure(8), we evaluate

$$
\left(\hat{\sigma}_{1} \hat{\sigma}_{2}^{-1}\right)^{3}|0,0,0\rangle=-\frac{1}{2}(|0,0,0\rangle+|0,1,1\rangle+|1,0,1\rangle+|1,1,0\rangle) .
$$

This state is entanglement-equivalent, as discussed in Definition(II.3) to $\frac{1}{\sqrt{2}}(|0,0,0\rangle+|1,1,1\rangle)$, the tripartite analogue of a Bell state (GHZ state) as can be seen by use of the local transformation ${ }^{2}$ $\begin{aligned} & V=v \otimes v \otimes v \text { where } v=\frac{1}{\sqrt{2}}\left(\begin{array}{cc}1 & 1 \\ -1 & 1\end{array}\right) \\ & V \frac{1}{\sqrt{2}}(|0,0,0\rangle+|1,1,1\rangle)=-\frac{1}{2}(|0,0,0\rangle+|0,1,1\rangle+|1,0,1\rangle+|1,1,0\rangle) .\end{aligned}$

The geometric action of "removing" one link corresponds algebraically to $\sigma_{1} \sigma_{2} \sigma_{2}{ }^{-1} \sigma_{1}{ }^{-1} \sigma_{2} \sigma_{2}{ }^{-1}=I$ which has no effect on the initial non-entangled generic state - see Figure(9).

\section{CONCLUSIONS}

We have attempted to display a relation between quantum entanglement and the topological properties of links. With this in mind we gave an elementary introduction to the quantum entanglement of bipartite systems, illustrating by a simple example how a naïve extension to the tripartite case fails. Nevertheless, the attempt to mimic the partial trace approach of the bipartite definition suggests an analogy with properties of links, especially of the Borromean Rings in the tripartite case.

With that in mind, we introduced the topic of braid groups, which provide a description of links in general. Starting with the bipartite case, we showed that a unitary representation of the twostring braid group $B_{2}$ gives an analogy between the Hopf Link and a maximally entangled (Bell)

\footnotetext{
2 The same transformations have been used in a similar context by the authors of Reference[7]
} 
state. Use of a continuous unitary representation of $B_{2}$ gives intermediate values of entanglement. Proceeding to the $B_{3}$ case, we showed that the braid word description of the Borromean Rings does indeed produce (an equivalent of ) the maximally-entangled tripartite GHZ state.

The braid group approach provides an illustrative bridge between a geometric picture of entanglement and the algebraic description of the quantum state, which may prove of value in elucidating some of the properties of the tripartite, and higher, cases.

\section{REFERENCES}

This note has been designed to be read without references. I have however included in the following some seminal papers, and also some general online sites which contain references for further reading. The analogy with Borromean rings was explored independently by Kauffman and Lomonaco [6]; and equivalent unitary representations of the braid groups producing entanglement were given by Chen, Xue and Ge in reference [7]

[1] W. K. Wootters, Phys. Rev. Lett. 80, 2245 (1998).

[2] D. M. Greenberger, M. Horne, and A. Zeilinger, Bell's Theorem, Ed. M. Kafatos (Kluwer, Dordrecht), p.69 (1989).

[3] http://en.wikipedia.org/wiki/Braid_group, http://mathworld.wolfram.com/BraidGroup.html

[4] http://en.wikipedia.org/wiki/Emil_Artin

[5] http://en.wikipedia.org/wiki/James_Waddell_Alexander_II

[6] L.H. Kauffman and S.J. Lomonaco, New Journal of Physics, 4, 73.1 - 73.18, ,2002) and private communication.

[7] J-L. Chen, K. Xue and M-L. Ge, Phys. Rev. A 76, 042324 (2007).

\section{APPENDIX: SYMMETRY GROUPS, BRAID GROUPS AND HECKE ALGEBRA}

We summarize here the presentations of some of the groups discussed in the text.

\section{Symmetric Group $S_{n}$}

Generators: $\left\{s_{i}: i=1 \ldots n-1\right\}$

Relations: $s_{i}^{2}=I \quad s_{i} s_{j}=s_{j} s_{i}(|i-j| \geq 2) \quad s_{i} s_{i+1} s_{i}=s_{i+1} s_{i} s_{i+1}$

Matrix Representation: $s_{i}=1 \times \cdots \times s \times 1 \times 1$ where $s=\left[\begin{array}{ll}0 & 1 \\ 1 & 0\end{array}\right]$ replaces each element 1 
along the diagonal.

\section{Braid Group $B_{n}$}

Generators: $\left\{\sigma_{i}: i=1 \ldots n-1\right\}$

Relations: $\sigma_{i} \sigma_{j}=\sigma_{j} \sigma_{i}(|i-j| \geq 2) \quad \sigma_{i} \sigma_{i+1} \sigma_{i}=\sigma_{i+1} \sigma_{i} \sigma_{i+1}$

\section{Hecke algebra}

This is a $q$-deformation of $S_{n}$ and has the relations of $B_{n}$ plus an additional one: Generators: $\left\{\sigma_{i}: i=1 \ldots n-1\right\}$

Relations: $\sigma_{i} \sigma_{j}=\sigma_{j} \sigma_{i}(|i-j| \geq 2) \quad \sigma_{i}^{2}=(1-q) \sigma_{i}+q \quad \sigma_{i} \sigma_{i+1} \sigma_{i}=\sigma_{i+1} \sigma_{i} \sigma_{i+1}$ Matrix Representation (Burau): $\sigma_{i}=1 \times \cdots \times \sigma \times 1 \times 1$ where $\sigma=\left[\begin{array}{cr}1-q & q \\ 1 & 0\end{array}\right]$ replaces each element 1 along the diagonal.

\section{Quasi-symmetric group}

Generators: $\left\{\sigma_{i}: i=1 \ldots n-1\right\}$

Relations: $\sigma_{i} \sigma_{j}=\sigma_{j} \sigma_{i}(|i-j| \geq 2) \quad \sigma_{i}^{R}=I \quad \sigma_{i} \sigma_{i+1} \sigma_{i}=\sigma_{i+1} \sigma_{i} \sigma_{i+1}$ Matrix Representation : $\sigma_{i}=1 \times \cdots \times \sigma \times 1 \times 1$ where $\sigma=\left[\begin{array}{cc}1-q & q \\ 1 & 0\end{array}\right]$ and $(-q)^{R}=I$.

VIII. FIGURES

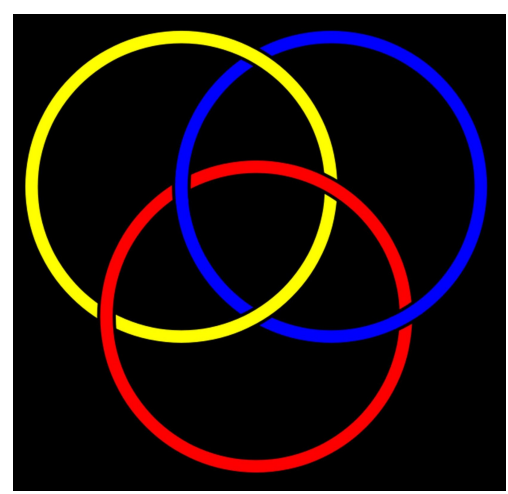

FIG. 1: Borromean Rings 


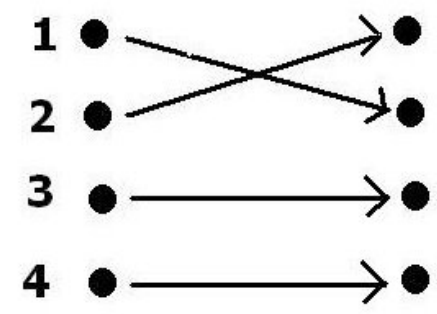

FIG. 2: An element of $S_{4}\left(s_{1}\right)$

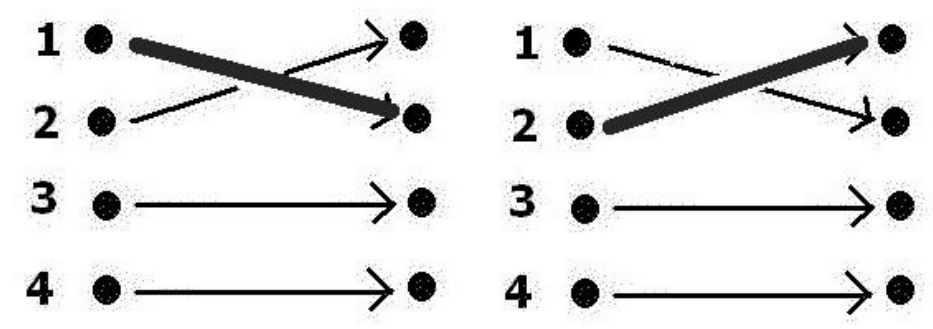

FIG. 3: $\sigma_{1}$ and $\sigma_{1}^{-1}$ of $B_{4}$

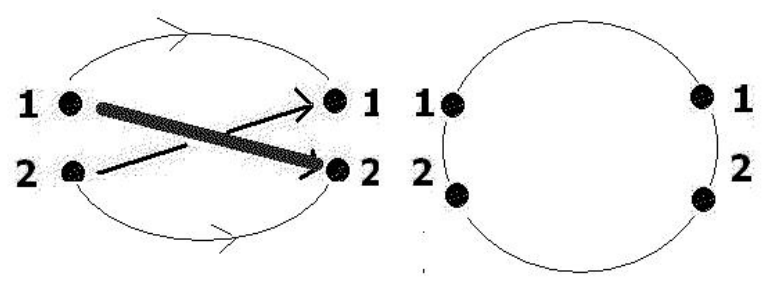

FIG. 4: In $B_{2}, \sigma_{1}$ produces the unknot 


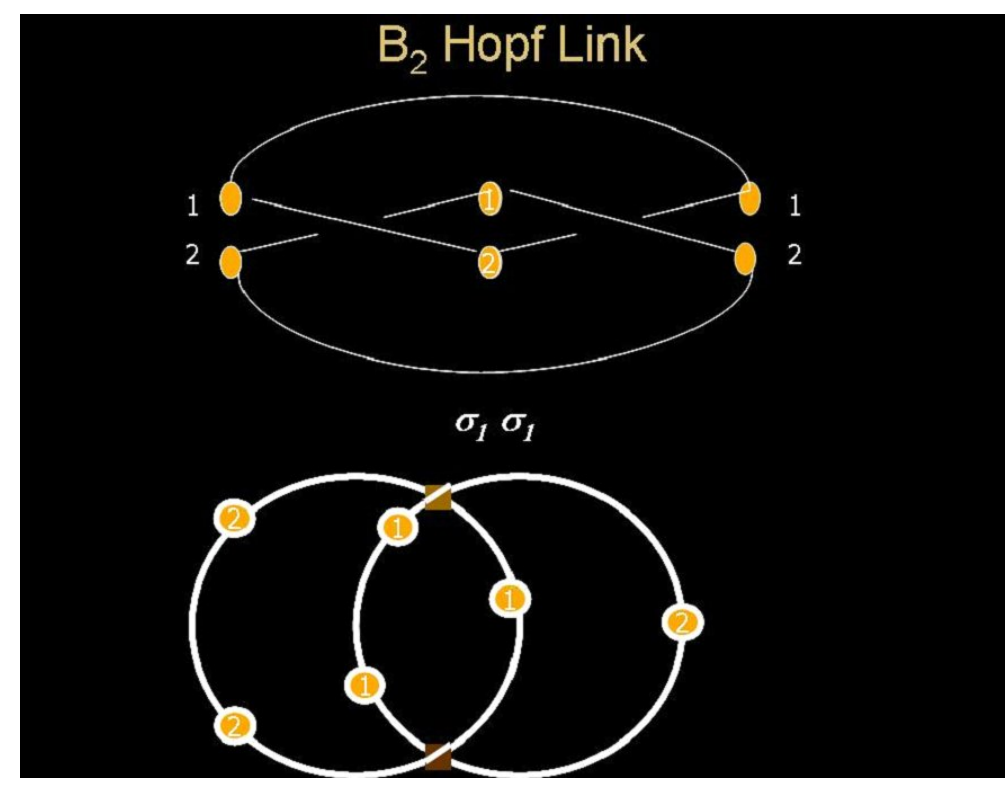

FIG. 5: In $B_{2}, \sigma_{1}^{2}$ produces the Hopf link

\section{More Complicated Example $\mathrm{B}_{5}$}

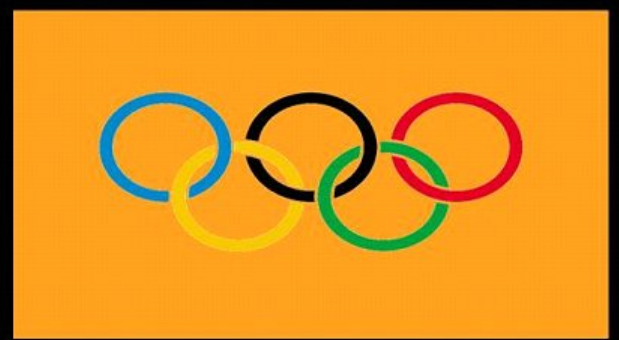

$B_{5}$

$$
\sigma_{1}^{2} \sigma_{2}^{2} \sigma_{3}^{2} \sigma_{4}^{2}
$$

FIG. 6: The Olympic Symbol in $B_{5}$ 
Entanglement v. Unitary Angle

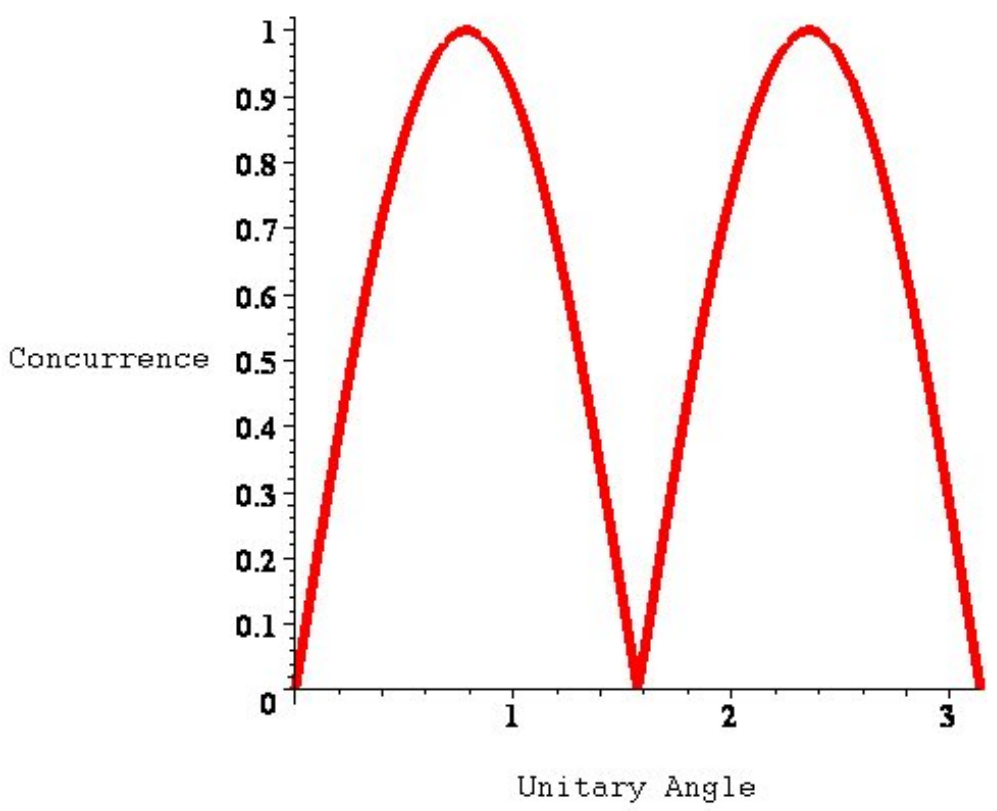

FIG. 7: Entanglement(Concurrence) v. Unitary Angle 0 to $\pi$

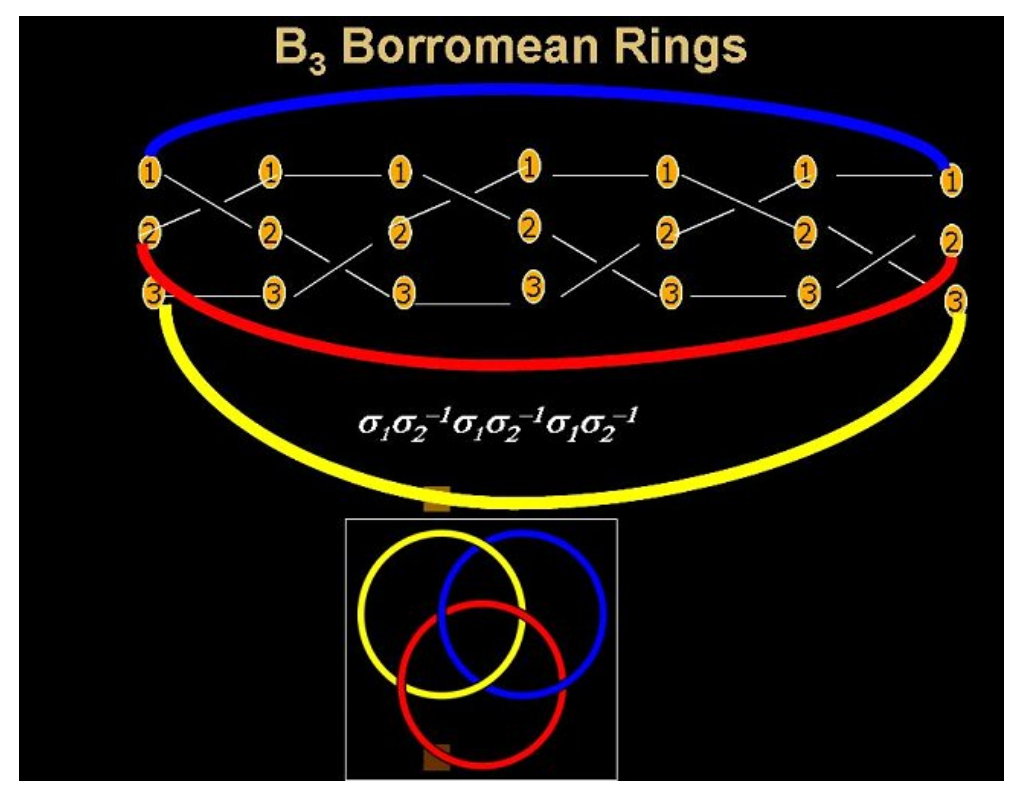

FIG. 8: Borromean Rings from $B_{3}$ 


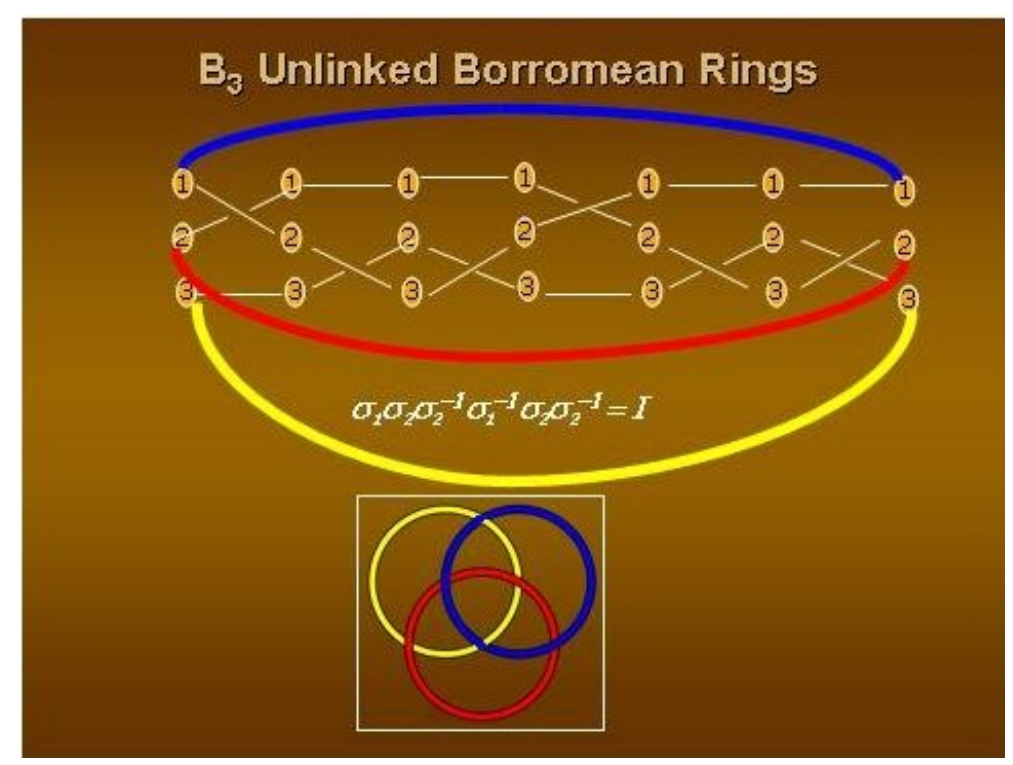

FIG. 9: Unlinked Borromean Rings from $B_{3}$ 